\title{
The Blossoming of 2D Materials
}

\author{
DUAN Xiangfeng ${ }^{1}, \mathrm{XU}$ Yang ${ }^{2}$ \\ ${ }^{1}$ Department of Chemistry and Biochemistry, UCLA, 607 Charles E. Young Drive East, Box 951569 Los Angeles, CA, USA. \\ ${ }^{2}$ College of Information Science \& Electronic Engineering, Zhejiang University, Hangzhou 310027, P. R. China. \\ Email: xduan@chem.ucla.edu (D.X.); yangxu-isee@zju.edu.cn (X.Y.). \\ Published online: May 30, 2019.
}

The vehement explosion in two-dimensional (2D) materials has led to one exciting new discoveries after another for the past 15 years. This circumstance resembles a famous movie "The Gold Rush" (1925) expressed the true story about the stream of American people as crowded as the hurricane to join the gold racing in the $19^{\text {th }}$ century. Similarly, the family of 2D materials has gained tremendous interest from broad scientific community in various fields, from fundamental chemistry and physics to materials and device engineering. This special issue includes both tutorial reviews and original research articles, providing general overviews of the recent progresses and bringing about the newest discoveries in this rapidly evolving field.

Controllable synthesis of $2 \mathrm{D}$ materials is foundational for realizing the extraordinary promises of $2 \mathrm{D}$ materials. The controllable, reproducible and scalable production of $2 \mathrm{D}$ materials represents a standing challenge of the field. The special issue dedicates a considerable fraction of spaces in the synthesis of various 2D materials. In a tutorial review, Liu et al. ${ }^{1}$ discussed the solution phase synthesis and processing of various 2D materials, heterostructures for the mass production of a variety of chemical functional-based heterostructures. In an original communication, Wu et al. ${ }^{2}$ reported a liquid-phase approach to antimonene quantum dots for applications in optical devices, molecular adsorption, and thermoelectric devices. Wang et al. ${ }^{3}$ reported a new synthesis method to three-dimensional (3D) graphene via biomass hydroxyapatite, offering a potential pathway to low-cost and mass production of $3 \mathrm{D}$ graphene for industrial applications. In addition, Chen et al. ${ }^{4}$ used the Kinetic Monte Carlo simulation to investigate the chemical vapor deposition (CVD) growth of $\mathrm{MoS}_{2}$, and found that the growth rate of second-layer is strongly depended on the first layer.

Defects play a critical role in determining the chemical, physical and electronic properties of 2D materials. Liu et al. ${ }^{5}$ reported a density functional theory (DFT) study to probe the role of point defects in modifying the electrical conductivity of graphene. They revealed breaking graphene honeycomb lattice by point defects lead to the localized states near the Fermi level to profoundly modify their electronic properties. Xiong et al. ${ }^{6}$ used molecular dynamics (MD) simulation to investigate the interaction of defective or doped graphene with polymer composite and their impacts on thermal conductivities. Additionally, Liu et al. ${ }^{7}$ systematically investigated the phonon vibration mode of single-layer to few layer and the bulk $\mathrm{WS}_{2}$ as a function of thickness and temperature.

Heterostructures are essential for creating functional devices for diverse applications. Li et al. ${ }^{8}$ gave a concise overview on the 2D van der Waals heterostructures and their potential applications for photodetection and other optoelectronic devices. Ren et al. ${ }^{9}$ reported the synthesis of $\mathrm{Ag} / \mathrm{WO}_{3-x}$ heterostructures that may enable rapid separation and transfer of photo generated charge carriers. In a tutorial review, Du et al. ${ }^{10}$ provided an overview of heterostructures formed between 2D materials and biomacromolecules, and showed specifically designed 2D material biomacromolecule heterostructures could lead to tailored electrical, mechanical, and biological characteristics. In another tutorial review, Cheng et al. ${ }^{11}$ discussed the potential in exploring nanoporous 2D materials for membrane separations with excellent flexibility, chemical stability, mechanical strength, and the ultimate atomic scale thickness.

Discovering and investigating new 2D materials is indispensible for continued expansion of the 2D material family, enabling improved properties or totally new functions. Zhang et al. ${ }^{12}$ reports a class of ferroelectric $2 \mathrm{D}$ materials $\left(\mathrm{ABP}_{2} \mathrm{X}_{6}\right)(\mathrm{A}$ : $\mathrm{Ag}, \mathrm{Cu}$; B: Bi, In; X: S, Se) with unique negative Posson's ratio, which may offer significant potential for the next-generation memory devices or nanoelectromechanical devices.

Together, we thank all the authors, referees and the editorial office for their efforts to make this special issue possible. We hope you all will enjoy this outstanding collection of research articles and tutorial reviews on the $2 \mathrm{D}$ blossoming.

\section{References}

(1) Liu, Q.; Wang, X. S.; Wang, J. L.; Huang, X. Acta Phys. -Chim. Sin. 2019, 35, 1099. doi: 10.3866/PKU.WHXB201811005

(2) Wu, H.; Yan, Z. Acta Phys. -Chim. Sin. 2019, 35, 1052. 
doi: 10.3866/PKU.WHXB201801262

(3) Wang, K. X.; Shi, L. R.; Wang, M. Z.; Yang, H.; Liu, Z. F.; Peng, H. L. Acta Phys. -Chim. Sin. 2019, 35, 1112. doi: 10.3866/PKU.WHXB201805032

(4) Chen, S.; Gao, J. F.; Srinivasan, B. M.; Zhang, Y. W. Acta Phys. -Chim. Sin. 2019, 35, 1119. doi: 10.3866/PKU.WHXB201812023

(5) Liu, N. S.; Zhou, S.; Zhao, J. J. Acta Phys. -Chim. Sin. 2019, 35, 1142. doi: 10.3866/PKU.WHXB201810040

(6) Xiong, Y. H.; Wu, H.; Gao, J. S.; Chen, W.; Zhang, J. C.; Yue, Y. N. Acta Phys. -Chim. Sin. 2019, 35, 1150. doi: 10.3866/PKU.WHXB201901002

(7) Liu, X. K.; Wang, J. L.; Xu, C. Y.; Luo, J. L.; Liang, D. S.; Cen, Y.
N.; Lü, Y. M.; Li, Z. W. Acta Phys. -Chim. Sin. 2019, 35, 1134. doi: 10.3866/PKU.WHXB201809013

(8) Li, J. Y.; Ding, Y.; Zhang, D. W.; Zhou, P. Acta Phys. -Chim. Sin. 2019, 35, 1058. doi: 10.3866/PKU.WHXB201812020

(9) Ren, Y. M.; Xu, Q. Acta Phys. -Chim. Sin. 2019, 35, 1157. doi: 10.3866/PKU.WHXB201812054

(10) Du, C. B.; Hu, X. L.; Zhang, G.; Cheng, Y. Acta Phys. -Chim. Sin. 2019, 35, 1078. doi: 10.3866/PKU.WHXB201812057

(11) Cheng, L.; Liu, G. P.; Jin, W. Q. Acta Phys. -Chim. Sin. 2019, 35, 1090. doi: 10.3866/PKU.WHXB201810059

(12) Zhang, C. M.; Nie, Y. H.; Du, A. J. Acta Phys. -Chim. Sin. 2019, 35, 1128. doi: 10.3866/PKU.WHXB201812037 